\title{
Study and Simulation of Transporting Radio Frequency Signals over Free Space Optics for Achieving High Data Rate and Power Saving
}

\author{
https://doi.org/10.3991/ijim.v15i16.24189 \\ Isam Aameer Ibrahim ${ }^{1,2(\varpi)}$, Tahreer Safa'a Mansour ${ }^{1}$ \\ ${ }^{1}$ University of Baghdad, Baghdad, Iraq \\ ${ }^{2}$ University of Thi-Qar, Nasiriyah, Iraq \\ isam.omair1101a@ilps.uobaghdad.edu.iq
}

\begin{abstract}
The remarkable technology for seamless integration of wireless and optical networks is radio frequency signals over Free Space Optics (FSO). This research study and simulation examine the design and evaluation performance of Radio Frequency over FSO (RF-FSO) wireless communication technology. These systems are implemented through medium communication link ranges to overcome excessive sensitivity of atmosphere medium and meet the requirements of a wide variety of optical wireless applications. There are two ways to achieve the application of the design mentioned above. The first way is the application of the Three modulation schemes of technology that is Amplitude shift keying (ASK), Quadrature amplitude modulation (QAM), and Quadrature phase-shift keying (QPSK) of digital modulation. The design of these modulation schemes is realized by using optiwave software to study the transmission of RF signals over the FSO channel and compare the three modulation techniques into the RF-FSO system. RF signals with the frequency range from (20 to 60) $\mathrm{GHz}$ is used in RF-FSO system and many carrier optical signals where the higher RF has a wider bandwidth to carrying larger information. To increase the transmission of data rates Wavelength Division Multiplexing (WDM) technology is used. The second way is based on a mathematical model which has been proposed for this study. This mathematical model calculates optimal beacon period (BI), and optimal listen interval (LI) to preventing the overlapping of time between the signals and the decrease in the required power. Using different weather conditions samples, the simulation results revealed that the best performance of the RF-FSO system is from link range, and the receiver is more sensitive. The simulation results are as follows: Two independent channels are transmitted 20 Gbps over ranges from $(263 \mathrm{~m}$ to $6.55 \mathrm{~km}$ ), while four channels are transmitted $40 \mathrm{Gbps}$ over ranges from $(257 \mathrm{~m}$ to $5.95 \mathrm{~km})$, and eight independent channels transmit $80 \mathrm{Gbps}$ over distance from $(203 \mathrm{~m}$ to $5.2 \mathrm{~km})$.
\end{abstract}

Keywords - free space optics, radio frequency, amplitude shift keying, quadrature amplitude modulation, quadrature phase shift keying, wavelength division multiplexing, power management 


\section{Introduction}

Wireless communication technology is widely used in today's world because of its flexibility and mobility. Due to these characteristics, the number of integrated and deployed applications into the wireless system is increased significantly. Numerous applications such as multimedia applications, real-time software, as well as control and monitor systems are operating on wireless networks [1]. One of the major issues of today's world revolves around power management for wireless networks. This is due to most of the devices that run on wireless technology are powered by a battery. Good power management will prolong battery life [2]. FSO is an optical wireless communication of transmission information from one point to another using an optical light source [3]. FSO communication utilizes the same rule and the same abilities of optical fiber with lower cost, but the optical light transitions through the atmosphere instead of the core of optical fiber to removing the constituting optical fiber and time [4]. FSO technology provides many advantages, such as immune to radio frequency interference, provide high data rate, low power, high security, low cost, and high bandwidth [5]. The radio wave is a wireless communication system used to transmitted data over the air by digital signal at a frequency range from $(3 \mathrm{kHz}$ to $300 \mathrm{GHz})$, the transmission being bi-directional between a mobile and an antenna [6]. Radio over Free Space Optics communication system consists of a transmitter, receiver, and an atmospheric medium (FSO) communication channel between them for transmission the signals which its passes through it [7]. The first transporting of modulating radio frequency (RF) signals through free space optics (Ro-FSO) by using analog transmitter and receiver and uses a couple of optical fiber into transmitter and receiver to control the signal through FSO link [8]. The range of transmitted signal was only 3 meters between telescope lenses of transmitter and receiver; wave division multiplexing has been investigated for transmitting two different wavelengths over free space optics [9]. A new advance of dense wavelength division multiplexing (DWDM) Ro-FSO communication technique has been used, which transport 2.5 Gbps over $1 \mathrm{~km}$ of two buildings, employed optical fiber onto both ends of buildings for transited and received the optical signal [10]. The DWDM has been developed, which transmitted 2.5 Gbps through a $1 \mathrm{~km}$ range of atmospheric turbulence between two buildings by transmitter and receiver antenna [11]. This research solution for creating Ro-FSO communication system with better performances, Ro-FSO is wireless communication networks consist of both FSO and radio over fiber (R-o-F) techniques utilize for removal (RF) signals overcrowding, by optical transmission wave over free space optics carrying RF signal without the need of using optical fiber. A gaussian optical filter placed during using WDM to separates the different transmitted wavelength thus filters for prevention the interference of received signal and power consumption is reduced.

\section{Literature review}

Radio frequencies over Free Space Optics (Ro-FSO) are modern technology that promises future wireless communications. Ro-FSO has widely spread because of 
the increased demand to have a wireless bandwidth [12]. Ro-FSO technology was studied due to many features like low attenuation losses, low power exhaustion, and high bandwidth for RF distribution [13]. Design and simulation of Ro-FSO wireless communication system offer an excessive sensitivity of atmosphere medium which causing fading in the received signal due to attenuation particles like (fog, rain, haze, snow, and smoke) that conflict with propagation beam leading to scattering and absorption that produce decreasing of received power. As well as, a line of sight (LOS) of the Ro-FSO system which needs to transmit the information directly to the receiver without any obstruction between them. Previews of research studies on Ro-FSO and some technologies used for wireless communication applications such as design and evaluation of a hybrid combination of Ro-FSO system used four different channels of both wavelength division multiplexing WDM and the mode division multiplexing MDM techniques, which each channel carried 2.5 Gbps placed on $10 \mathrm{GHz}$ radio frequency signals modulated by (MZM) with four optical beams for transmitting 10 Gbps data rate [14]. System design based on (DWDM) dense wavelength division multiplexing and focal scheme technologies that transmit $320 \mathrm{Gbps}$ through free-space optical (FSO), therefore has been employed eight optical channels for transmission $320 \mathrm{Gbps}$ a long 50 meters of FSO system. They have produced that increase of data rate by DWDM and focal scheme [15]. Designed a simulation system of a hybrid orthogonal frequency division multiplexing (OFDM) Ro-FSO communication system had (MDM) mode division multiplexing of two independent channels, thus capable for transmissions 40 Gbps which each channel transport 20 Gbps that carried by radio frequency (RF) signals [16]. As for the Ro-FSO system, it offers an attractive and promising way to address the study of the fluctuations of an optical signal that occur under different weather conditions through free-space optics, and it able of transporting electrical modem through FSO it should be placed onto optical source after carrying the information by amplitude, frequency, or phase modulation of RF signal.

\section{Design two channels of Ro-FSO system}

This design has a Quadurater Phase shift keying (QPSK) modulator where the incoming bit rate has split into two sequences signal. The phase of a signal has to change according to the input binary bit symbol. QPSK modulator block contains, as shown in Figure 1, a PSK pulse generator, and a quadrature modulator, a PSK pulse generator consisting of a PSK sequence generator, and two M-ray pulse generators. This gives two signals with different phases by one digit, inputs quadrature modulator multiplexing by cosine and sine carrier wave that gathers by an adder to produce output electrical signal. QAM is a form of hybrid modulation that has been used to code and modulate in this system QAM pulse generator consisting of sequence generator and two M-ray pulse generators. The input bit rate is divided into two subsequences; this generated signal pulse varies amplitude according to the input bits symbol. The two pulses multiple by two carrier signal sine and cosine of quadrature modulator they adding together for output an electrical modulated signal. 


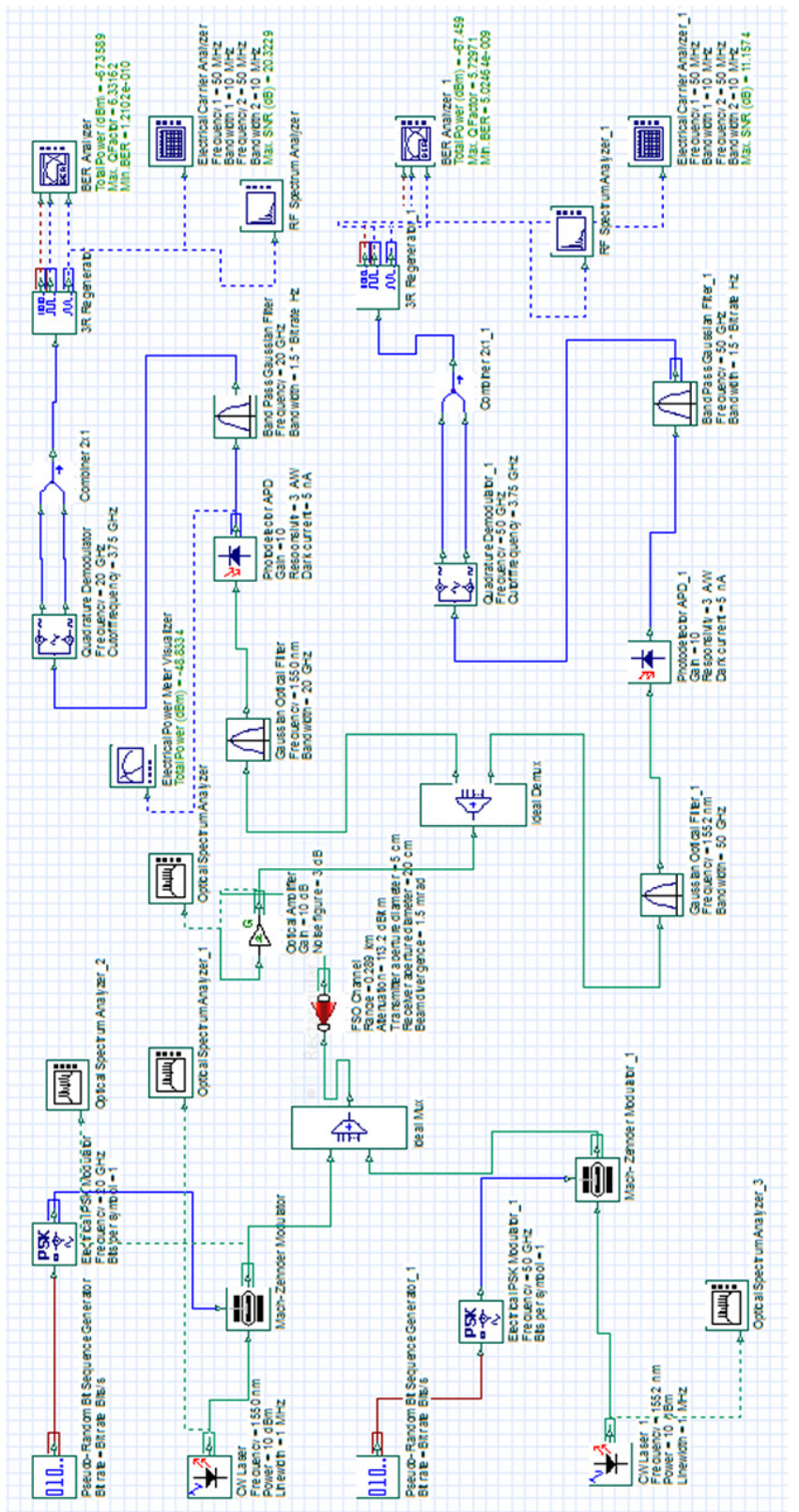

Fig. 1. Two channels Ro-FSO system

\section{Design four channels of Ro-FSO system by using WDM}

In this section, WDM has been used to develop the simulation system of a digital modulator (QPSK) from single to multi-channel in the Ro-FSO system. At the receiver, 
De-WDM uses to distribute signals onto the receiving channels. To avoid the interference at the receiver its must place a Gaussian optical filter after De-WDM to separate the receiving wavelengths according to transmitted waves. These systems have been using the ideal WDM, which is different from normal WDM; it has no filters inside.

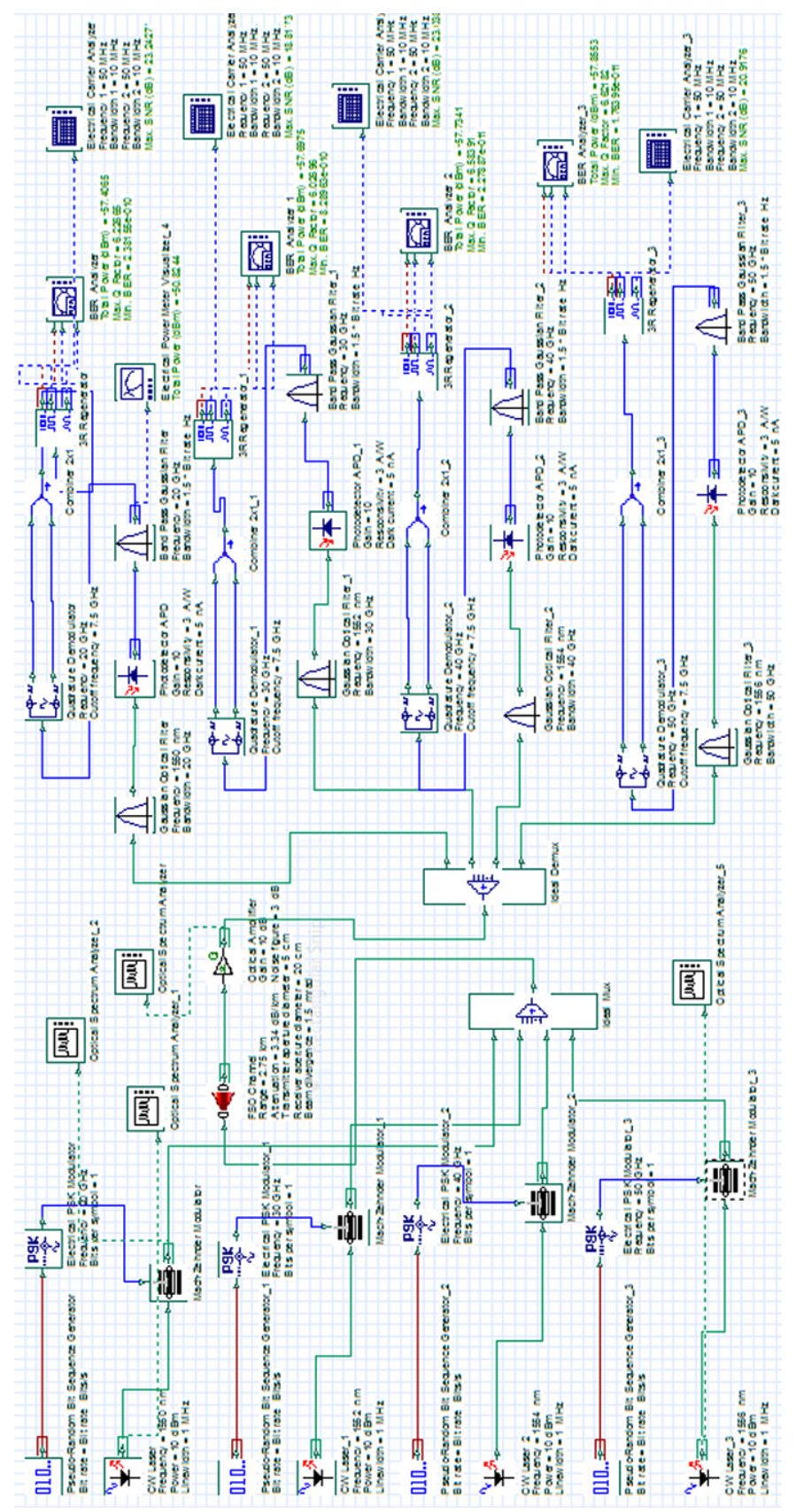

Fig. 2. Four channels WDM Ro-FSO system 


\section{$5 \quad$ Design eight channels of Ro-FSO system by using WDM}

The architectural design of the eight channels of the Ro-FSO system is similar to that of the four channels of the Ro-FSO system, but Pseudo-Random Binary Sequences (PRBS) could be transmission by $8 \lambda$ system design.

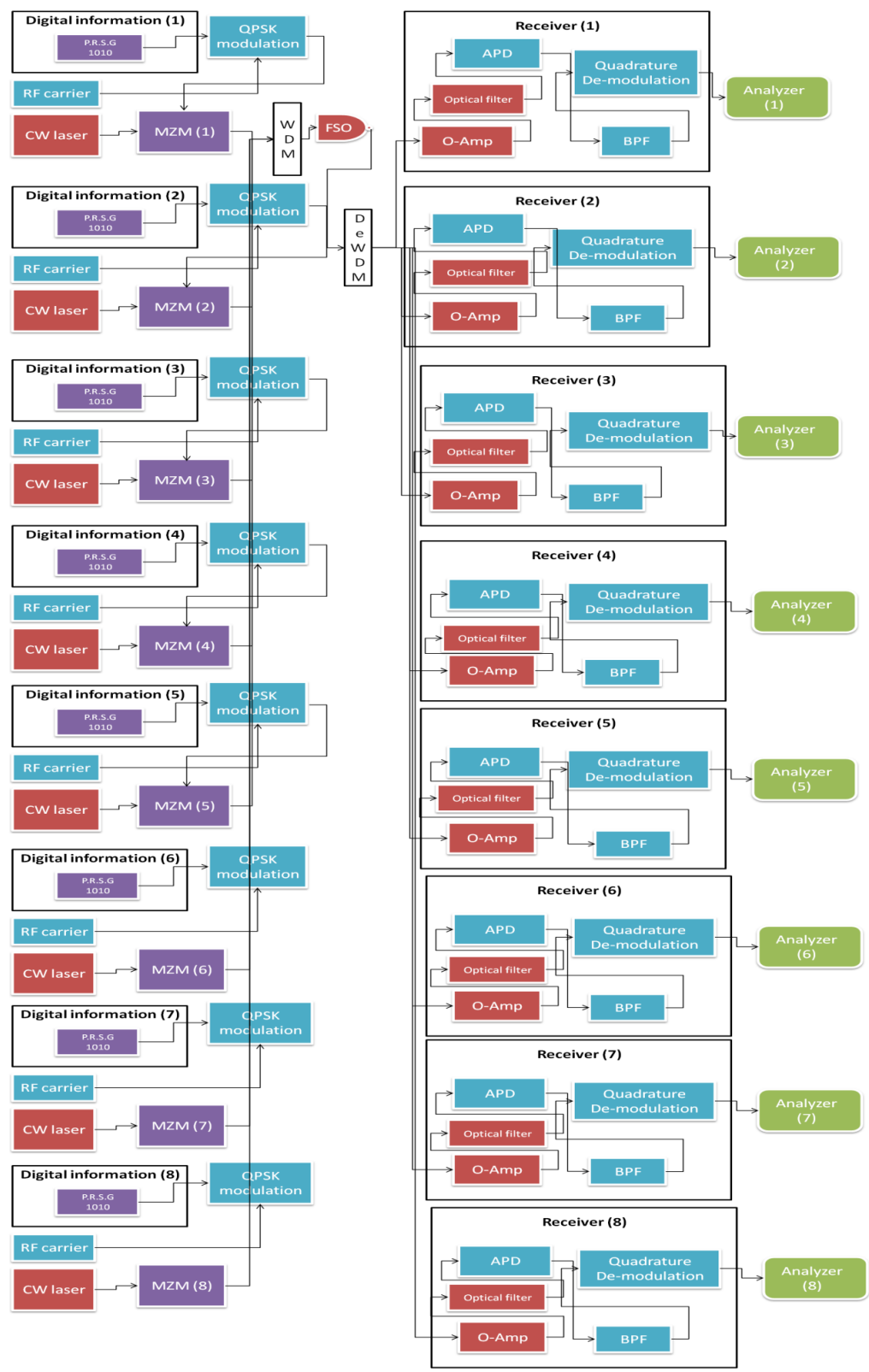

Fig. 3. Eight channels WDM Ro-FSO system 
Table 1. The parameters of Ro-FSO simulation system design

\begin{tabular}{|l|c|c|}
\hline \multicolumn{1}{|c|}{ Parameter } & Value & Unit \\
\hline Bit rate & 10 & Gbps \\
\hline CW laser & 1550 & $\mathrm{~nm}$ \\
\hline Transmission power & 10 & $\mathrm{dBm}$ \\
\hline Transmitter aperture diameter & 5 & $\mathrm{~cm}$ \\
\hline Receiver aperture diameter & 20 & $\mathrm{~cm}$ \\
\hline Beam divergence & 1.5 & $\mathrm{mrad}$ \\
\hline Gain of an optical amplifier & 10 & $\mathrm{~dB}$ \\
\hline Noise figure & 3 & $\mathrm{~dB}$ \\
\hline Gain of APD & 10 & \\
\hline Responsively of APD & 3 & $\mathrm{~A} / \mathrm{W}$ \\
\hline The dark current of APD & 5 & $\mathrm{nA}$ \\
\hline
\end{tabular}

Table 2. Values of free space attenuation parameters employed in simulation systems

\begin{tabular}{|l|c|l|}
\hline \multicolumn{1}{|c|}{ W. C } & $\alpha(\mathrm{dB} / \mathrm{km})$ & $c_{n^{2}}$ \\
\hline Clear air & 0.43 & $5 \times 10^{-14}$ \\
\hline Haze & 3.34 & $1.7 \times 10^{-14}$ \\
\hline Light rain & 1.98 & $0.6 \times 10^{-14}$ \\
\hline Mod rain & 5.84 & $0.5 \times 10^{-14}$ \\
\hline Heavy rain & 9.29 & $0.4 \times 10^{-14}$ \\
\hline Light fog & 16.67 & $0.3 \times 10^{-14}$ \\
\hline Mod fog & 35.38 & $0.2 \times 10^{-14}$ \\
\hline Heavy fog & 113.2 & $0.1 \times 10^{-14}$ \\
\hline
\end{tabular}

Every channel generates 10 Gbps by PRBS generator. The values of weather conditions of Table 2 that was used for test performances of Ro-FSO systems designs are taken from ref [17].

\section{Mathematical model of power saving mode}

The purpose of implementing the proposed analysis is to determine a precise average ratio of the time when the station remains in the doze mode. This approach aims to reduce the inefficiency of two factors found in the Power Saving Mode (PSM) mechanism, namely, channel contention and prevention of frequent waking up of stations. There are two random variables in this proposed scheme: interframe arrival time and packet frame size. The interframe arrival time can be expressed in three types of distributions; deterministic (DET), uniform (UNI), and exponential (EXP). Meanwhile, the size of the packet frame distribution is either uniform or deterministic in distribution. For an energy-saving scheme to work, the network must not be in a state of heavy load. This is because the PSM mechanism operates optimally when the network utilization ( $\rho$ ) is equal to or less than $30 \%$ [18-20]. Therefore, in this research analysis, the benchmark 
of the utilization value must be equal to or less than $30 \%$ for every element. As such, the calculation of the utilization value is based on the formula of utilization as below.

$$
\rho=S \times \sum_{J=1}^{c} \frac{1}{U}
$$

Where $\mathrm{S}$ is the communication time with consideration of the actual transmission time from the Optical Channel (OC) to the element located at the receiver and to the Power Save (PS-POLL) frame, with acknowledgment of the frame from the element to the free space optics channel that is free of channel contention, the value of the communication time must be higher than the value of actual transmission between the optical channel and the elements located at the receiver. This is because the PSM mechanism has sleeping periods and the presence of buffered packet frames at the optical channel, which need more control during the sending of packet frames to the elements. By using the PSM mechanism, it avoids packet drop and channel contention; both of them consume more energy.

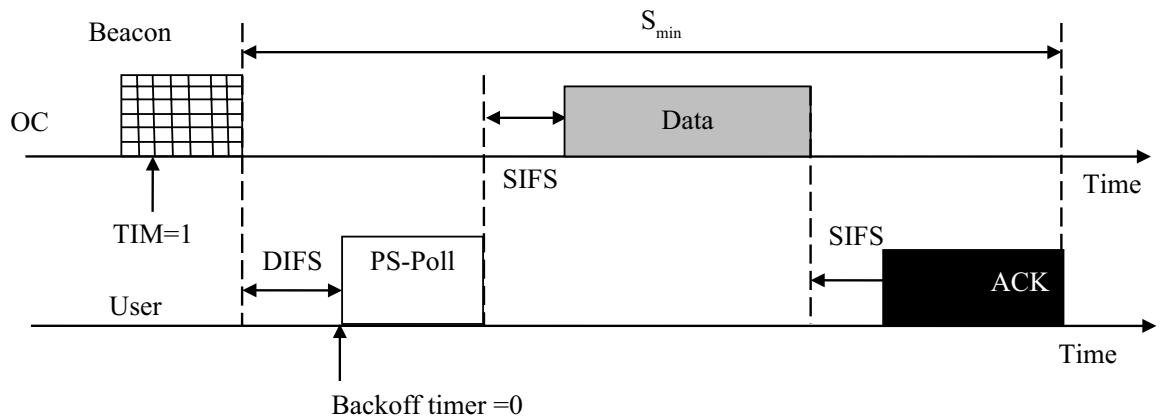

Fig. 4. The shortest communication time of receiving one packet frame

Figure 4 shows the shortest communication time for retrieving one data frame from an $\mathrm{OC}$ to the element and the transmission time of three frames. After receiving one beacon frame, the PSM-enabled user must wait for a short period for the Distribution Coordinate Function Interframe Space (DIFS) to buffer the packet frames before sending the PS-POLL frame to the OC. Then, the station sends out PS-POLL frames to the $\mathrm{OC}$ after waiting for a random backoff timer that changes from $(0)$ to the current contention window $(\mathrm{CW})$. The OC responds to the PS-POLL frame immediately by transmitting the packet frames after sending the shortest period namely, Short Interframe Space (SIFS). Only when the PSM-enabled user has received this packet frame successfully, the PSM-enabled station performs the task of sending the ACK frame to the OC after the SIFS time is released from the same station to the OC [19-23]. Based on the Distribution Coordinate Function (DCF) [20], the formula for the calculation of the communication time is as follows:

$$
\mathrm{S}_{\mathrm{act}}=\frac{\mathrm{PDS}}{\mathrm{DTR}}+\frac{\mathrm{PFS}}{\mathrm{BTR}}+\frac{\mathrm{ACK}}{\mathrm{BTR}}+\mathrm{CW}+\mathrm{DIFS}+3 \mathrm{SIFS}=1.139 \mu \mathrm{s}
$$


Table 3. Parameters simulation that is used to calculate $\mathrm{S}$ [24]

\begin{tabular}{|l|c|}
\hline \multicolumn{1}{|c|}{ Parameters } & Values \\
\hline Number of stations & 1 to 10 \\
\hline Data Transmission Rate (DTR) & $11 \mathrm{Mbps}$ \\
\hline Basic Transmission Rate (BTR) & $2 \mathrm{Mbps}$ \\
\hline Size of Packet Data (PDS) & $6000 \mathrm{bits}$ \\
\hline Size of PS-POLL frame (PFS) & 14 bytes \\
\hline Size of ACK frame (ACK) & 14 bytes \\
\hline Interframe Space-Time (SIFS) & $10 \mu \mathrm{s}$ \\
\hline Distribution of Interframe Space (DIFS) & $50 \mu \mathrm{s}$ \\
\hline Minimum Contention Window (MCW) & 31 \\
\hline Preamble & $192 \mu \mathrm{s}$ \\
\hline
\end{tabular}

\section{$7 \quad$ Results and discussion}

The results of the Ro-FSO communication systems are extracted using "OptiSystem" software to improve the performance of the single-channel Ro-FSO, and multi-channel WDM Ro-FSO systems. In the two channels of the Ro-FSO system, two optical waves $(1550,1552) \mathrm{nm}$ are transmitted. Each one is carrying $10 \mathrm{Gbps}$, one placed onto $20 \mathrm{GHz}$ of RF signal transmission by $1550 \mathrm{~nm}$ laser source and the other onto signal $50 \mathrm{GHz}$ traveled by $1552 \mathrm{~nm}$. The power measurements of the transmitted signal and received signal, after $263 \mathrm{~m}$ range under heavy fog weather conditions by the optical spectrum analyzer (OSA), were $10 \mathrm{dBm}$ and $-26.707 \mathrm{dBm}$.

Table 4. Results of 2-channels transfer 20 Gbps over maximums range [25]

\begin{tabular}{|c|c|c|c|c|c|c|}
\hline $\begin{array}{c}\text { Weather } \\
\text { Conditions }\end{array}$ & $\alpha(\mathrm{dB} / \mathrm{km})$ & $c_{n^{2}}$ & Max Link & RF(GHz) & BER & Q.factor \\
\hline \multirow[t]{2}{*}{ Clear air } & \multirow[t]{2}{*}{0.43} & \multirow[t]{2}{*}{$5 \times 10^{-14}$} & \multirow[t]{2}{*}{$6.55 \mathrm{~km}$} & 20 & $7.9 \times 10^{-10}$ & 6.03 \\
\hline & & & & 50 & $1.8 \times 10^{-10}$ & 6.26 \\
\hline \multirow[t]{2}{*}{ Light fog } & \multirow[t]{2}{*}{16.67} & \multirow[t]{2}{*}{$0.3 \times 10^{-14}$} & \multirow[t]{2}{*}{$1.09 \mathrm{~km}$} & 20 & $7.8 \times 10^{-10}$ & 6.03 \\
\hline & & & & 50 & $1.8 \times 10^{-10}$ & 6.26 \\
\hline \multirow[t]{2}{*}{ Mod fog } & \multirow[t]{2}{*}{35.38} & \multirow[t]{2}{*}{$0.2 \times 10^{-14}$} & \multirow[t]{2}{*}{$0.641 \mathrm{~km}$} & 20 & $9.4 \times 10^{-10}$ & 6.00 \\
\hline & & & & 50 & $2.5 \times 10^{-10}$ & 6.21 \\
\hline \multirow[t]{2}{*}{ Heavy fog } & \multirow[t]{2}{*}{113.2} & \multirow[t]{2}{*}{$0.1 \times 10^{-14}$} & \multirow[t]{2}{*}{$0.263 \mathrm{~km}$} & 20 & $8.3 \times 10^{-10}$ & 6.025 \\
\hline & & & & 50 & $2.0 \times 10^{-10}$ & 6.25 \\
\hline
\end{tabular}

Figure 5 shows the results of the processes in Table 4 . The red lines demonstrate transmissions of $20 \mathrm{Gbps}$ when different radio frequency signals (20 and 50) $\mathrm{GHz}$ carry $10 \mathrm{Gbps}$ that travel through the longest possible distance of FSO. The purple curves line explains transmissions of $10 \mathrm{Gbps}$. The less bit rate of $10 \mathrm{Gbps}$ could travel over the longest range than $20 \mathrm{Gbps}$ information under the four weather conditions. 


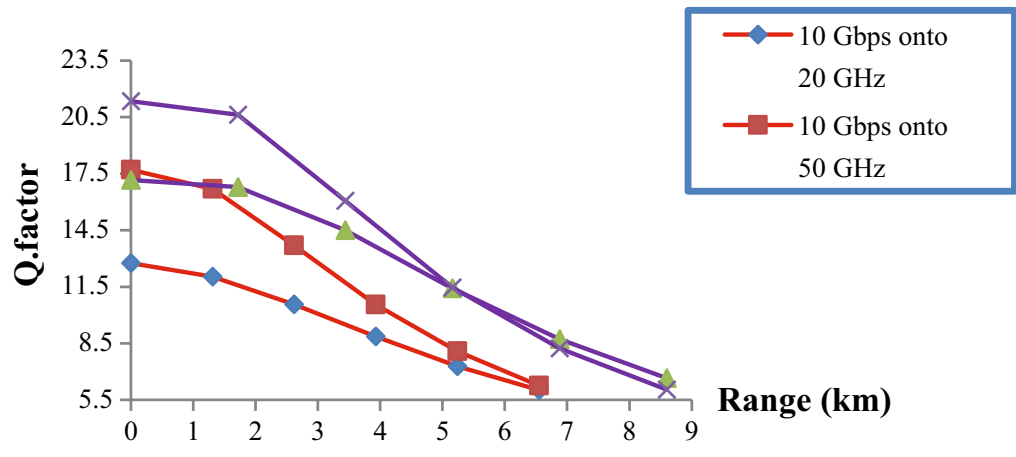

Fig. 5. Transporting 10 and 20 Gbps by 2-channel Ro-FSO under clear air

In the four channels Ro-FSO system, the information 40 Gbps has been transmissions via free-space optics by different signals of radio frequencies $(20,30,40,50) \mathrm{GHz}$ where every signal is carrying $10 \mathrm{Gbps}$ of information. The RF signals modulated with laser sources $(1550,1552,1554,1556) \mathrm{nm}$ the different wavelength mixing by WDM. Optical spectrum analyzer (OSA) measured the power of transmissions modulation signals before and after $255 \mathrm{~m}$ of FSO range under heavy fog weather conditions. The powers were $13.01 \mathrm{dBm}$ and $-22.55 \mathrm{dBm}$, respectively, after the longest range can be in 4-channels WDM Ro-FSO communication design. Table 5 shows the results of transmission 40 Gbps over maximum ranges can obtain under attenuated and turbulence weather by haze and rainy conditions. These results have been observed that a higher RF has a better performance which the signal RF $50 \mathrm{GHz}$ modulated with $1556 \mathrm{~nm}$ has a higher Q.factor and lower BER in all weather conditions that have been tested [26-27].

Table 5. Results of transporting 40 Gbps by 4-channels WDM Ro-FSO over the max link

\begin{tabular}{|c|c|c|c|c|c|c|}
\hline $\begin{array}{l}\text { Weather } \\
\text { Conditions }\end{array}$ & $\alpha(\mathrm{dB} / \mathbf{k m})$ & $c_{n^{2}}$ & Max Link & RF(GHz) & Q.factor & BER \\
\hline \multirow[t]{4}{*}{ Haze } & \multirow[t]{4}{*}{3.34} & \multirow[t]{4}{*}{$1.7 \times 10^{-14}$} & \multirow[t]{4}{*}{$2.75 \mathrm{~km}$} & 20 & 6.22 & $2.3 \times 10^{-10}$ \\
\hline & & & & 30 & 6.02 & $8.2 \times 10^{-10}$ \\
\hline & & & & 40 & 6.58 & $2.2 \times 10^{-10}$ \\
\hline & & & & 50 & 6.62 & $1.7 \times 10^{-11}$ \\
\hline \multirow[t]{4}{*}{ Light rain } & \multirow[t]{4}{*}{1.98} & \multirow[t]{4}{*}{$0.6 \times 10^{-14}$} & \multirow[t]{4}{*}{$3.5 \mathrm{~km}$} & 20 & 6.32 & $1.2 \times 10^{-10}$ \\
\hline & & & & 30 & 6.12 & $4.9 \times 10^{-10}$ \\
\hline & & & & 40 & 6.70 & $1.0 \times 10^{-11}$ \\
\hline & & & & 50 & 6.73 & $7.9 \times 10^{-12}$ \\
\hline \multirow[t]{4}{*}{ Mod rain } & \multirow[t]{4}{*}{5.84} & \multirow[t]{4}{*}{$0.5 \times 10^{-14}$} & \multirow[t]{4}{*}{$2 \mathrm{~km}$} & 20 & 6.35 & $1.0 \times 10^{-10}$ \\
\hline & & & & 30 & 6.15 & $3.7 \times 10^{-10}$ \\
\hline & & & & 40 & 6.73 & $8.2 \times 10^{-12}$ \\
\hline & & & & 50 & 6.76 & $6.4 \times 10^{-12}$ \\
\hline
\end{tabular}


Table 5. Results of transporting 40 Gbps by 4-channels WDM Ro-FSO over the max link (Continued)

\begin{tabular}{|l|c|c|c|c|c|c|}
\hline $\begin{array}{c}\text { Weather } \\
\text { Conditions }\end{array}$ & $\alpha(\mathrm{dB} / \mathrm{km})$ & $c_{n^{2}}$ & Max Link & $\mathrm{RF}(\mathrm{GHz})$ & Q.factor & BER \\
\hline Heavy rain & 9.29 & $0.4 \times 10^{-14}$ & $1.52 \mathrm{~km}$ & 20 & 6.30 & $1.4 \times 10^{-10}$ \\
\cline { 4 - 7 } & & & & 30 & 6.09 & $4.2 \times 10^{-10}$ \\
\cline { 4 - 7 } & & & & 40 & 6.66 & $1.2 \times 10^{-11}$ \\
\cline { 4 - 7 } & & & & 50 & 6.70 & $1.0 \times 10^{-11}$ \\
\hline
\end{tabular}

Figure 6 explains the best RF signals of transporting 10 Gbps through FSO link by curves under haze weather conditions, where the carrier signal $50 \mathrm{GHz}$ shown a higher Q.factor with max range. The max range $(2.75 \mathrm{~km})$ and attenuation of haze have been fixed.

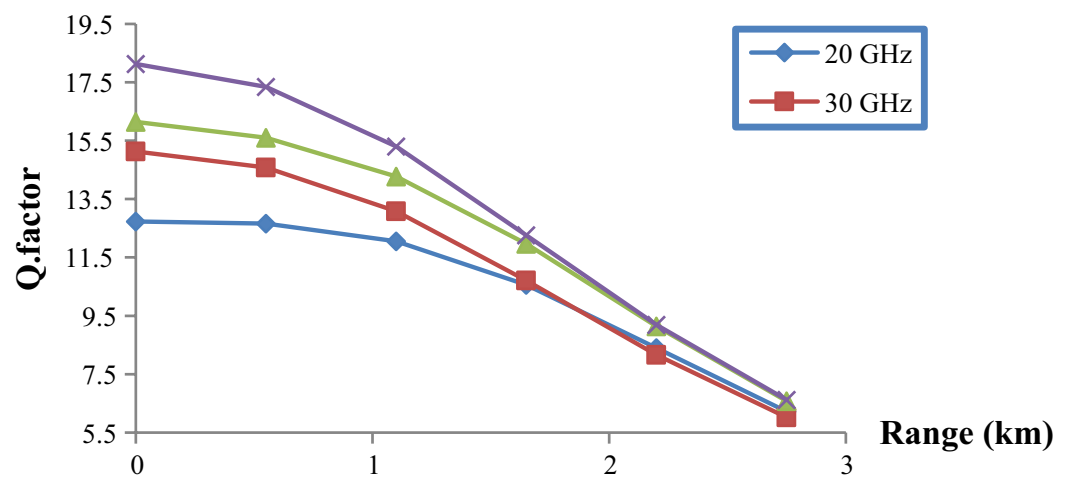

Fig. 6. The Q.factor of transporting 40 Gbps by 4-channels (WDM) Ro-FSO under haze weather conditions

In the eight channels of the Ro-FSO system, the information 80 Gbps has been transmissions via free-space optics by different signals of radio frequencies. The eight 10 Gbps pleased eight of $20 \mathrm{GHz}$ RF signals that have been optically modulating with different optical sources from $(1552.5,1551.7,1550.9,1550.2,1549.3,1548.5,1547.7$, 1546.9) $\mathrm{nm}$ by eight Mach-Zender-Modulator. The optical power of wavelengths before and after transported the information along a maximum range of $200 \mathrm{~m}$ has been determined by an optical spectrum analyzer under heavy fog weather conditions. The optical signal transmissions were $16.02 \mathrm{dBm}$ and $-11.47 \mathrm{dBm}$, the power before and after the $200 \mathrm{~m}$ range through heavy fog atmosphere attenuation.

Table 6 shows results of the longest distance that information $80 \mathrm{Gbps}$ could be reached under three weather conditions. The RF signals that have been used were only $20 \mathrm{GHz}$ which each $20 \mathrm{GHz}$ modulated with different laser wavelengths and has to mix with the same ideal WDM that has been used above systems. However, the signal has the furthest distances $(5 \mathrm{~km})$ under clear air and $(1.2 \mathrm{~km})$ under heavy rain, this due to fewer attenuation particles in clear air made signal capable of transmitting long ranges, and when attenuation increased this lead to a decrease the transmission range in heavy rain, while the heavy fog weather the range that signal could have its only $(200 \mathrm{~m})$ because FSO high sensitive to fog particles [23]. 
Table 6. Results of transporting 80 Gbps by ideal WDM in Ro-FSO system

\begin{tabular}{|c|c|c|c|c|c|c|}
\hline $\begin{array}{c}\text { Weather } \\
\text { Conditions }\end{array}$ & $\alpha(\mathrm{dB} / \mathrm{km})$ & $c_{n^{2}}$ & $\begin{array}{l}\text { Max } \\
\text { Link }\end{array}$ & $\begin{array}{c}\text { Laser Source } \\
(\mathbf{n m})\end{array}$ & Q.factor & BER \\
\hline \multirow[t]{8}{*}{ Clear air } & \multirow[t]{8}{*}{0.43} & \multirow[t]{8}{*}{$5 \times 10^{-14}$} & \multirow[t]{8}{*}{$5 \mathrm{~km}$} & 1552.5 & 7.48 & $3.5 \times 10^{-14}$ \\
\hline & & & & 1551.7 & 7.25 & $1.9 \times 10^{-13}$ \\
\hline & & & & 1550.9 & 7.18 & $3.3 \times 10^{-13}$ \\
\hline & & & & 1550.2 & 7.63 & $1.0 \times 10^{-14}$ \\
\hline & & & & 1549.3 & 7.04 & $9.2 \times 10^{-13}$ \\
\hline & & & & 1548.5 & 7.37 & $8.4 \times 10^{-14}$ \\
\hline & & & & 1547.7 & 6.29 & $1.5 \times 10^{-10}$ \\
\hline & & & & 1546.9 & 7.24 & $2.1 \times 10^{-13}$ \\
\hline \multirow[t]{8}{*}{ Heavy rain } & \multirow[t]{8}{*}{9.29} & \multirow[t]{8}{*}{$0.4 \times 10^{-14}$} & \multirow[t]{8}{*}{$1.2 \mathrm{~km}$} & 1552.5 & 9.08 & $5.2 \times 10^{-20}$ \\
\hline & & & & 1551.7 & 8.80 & $6.3 \times 10^{-19}$ \\
\hline & & & & 1550.9 & 8.72 & $1.3 \times 10^{-18}$ \\
\hline & & & & 1550.2 & 9.72 & $1.2 \times 10^{-22}$ \\
\hline & & & & 1549.3 & 8.87 & $3.4 \times 10^{-19}$ \\
\hline & & & & 1548.5 & 8.66 & $2.3 \times 10^{-18}$ \\
\hline & & & & 1547.7 & 7.50 & $3.1 \times 10^{-14}$ \\
\hline & & & & 1546.9 & 8.69 & $1.6 \times 10^{-18}$ \\
\hline \multirow[t]{8}{*}{ Heavy fog } & \multirow[t]{8}{*}{113.2} & \multirow[t]{8}{*}{$0.1 \times 10^{-14}$} & \multirow[t]{8}{*}{$200 \mathrm{~m}$} & 1552.5 & 10.18 & $1.1 \times 10^{-24}$ \\
\hline & & & & 1551.7 & 9.87 & $2.7 \times 10^{-23}$ \\
\hline & & & & 1550.9 & 9.85 & $3.2 \times 10^{-23}$ \\
\hline & & & & 1550.2 & 11.40 & $1.9 \times 10^{-30}$ \\
\hline & & & & 1549.3 & 10.32 & $2.6 \times 10^{-25}$ \\
\hline & & & & 1548.5 & 9.57 & $5.2 \times 10^{-22}$ \\
\hline & & & & 1547.7 & 8.65 & $2.3 \times 10^{-18}$ \\
\hline & & & & 1546.9 & 9.72 & $1.1 \times 10^{-22}$ \\
\hline
\end{tabular}

Figure 7 demonstrates the best transmissions wavelength of different wavelengths where the $(1550 \mathrm{~nm})$ wavelength has the best performances under the three weather conditions tested in this design.

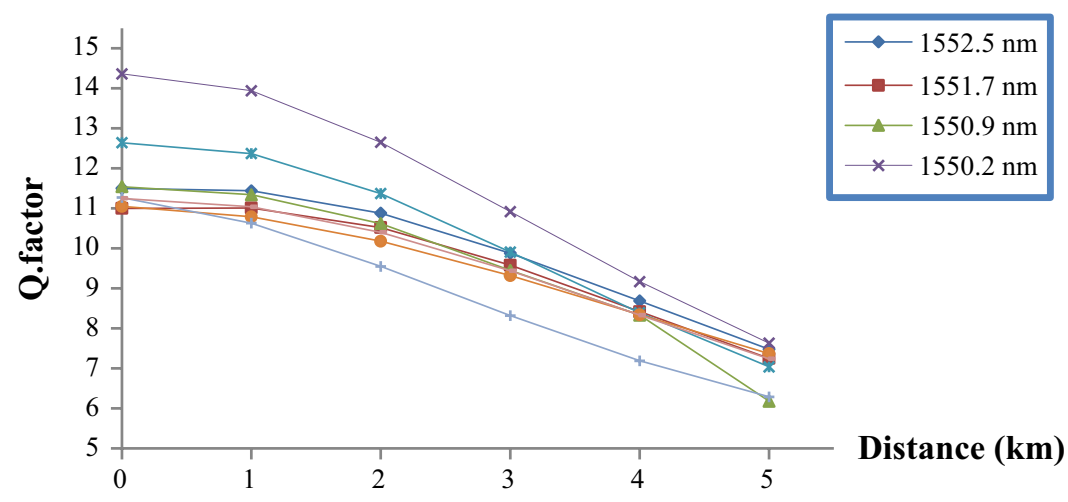

Fig. 7. The Q.factor of transporting $80 \mathrm{Gbps}$ by 8 -channels under clear air 
The optimal beacon interval (BI) and listen interval (LI) are calculated using Microsoft Visual C++ 2010 and the least common multiple. The calculation between the free space optic channel and the users achieves the value of optimal LI is $108 \mathrm{~ms}$ with average delay value, which yields several $60 \mathrm{~ms}$ for each packet. As shown in Figure 8, the value of average packet delay increases when LI increases.

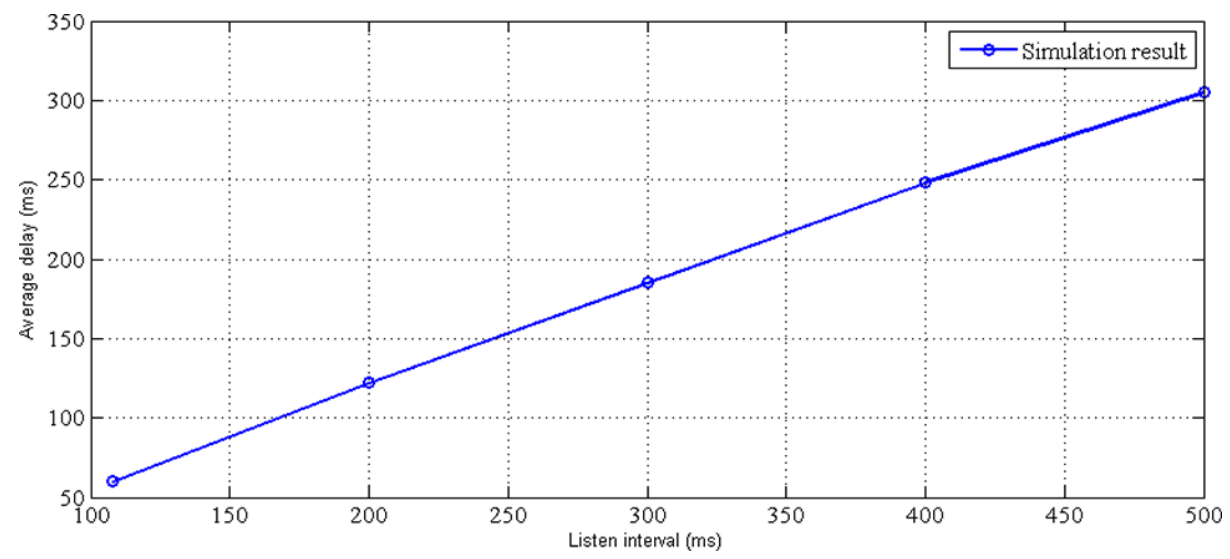

Fig. 8. Relation between average packet delays with values of LI

The calculation between the free space optic channel and the users achieves the value of optimal BI is $85 \mathrm{~ms}$ with a value of buffered packet frames delay is $52 \mathrm{~ms}$. The analysis in this research shows that value of buffered packet delay increase when the value of BI increases, as depicted in Figure 9.

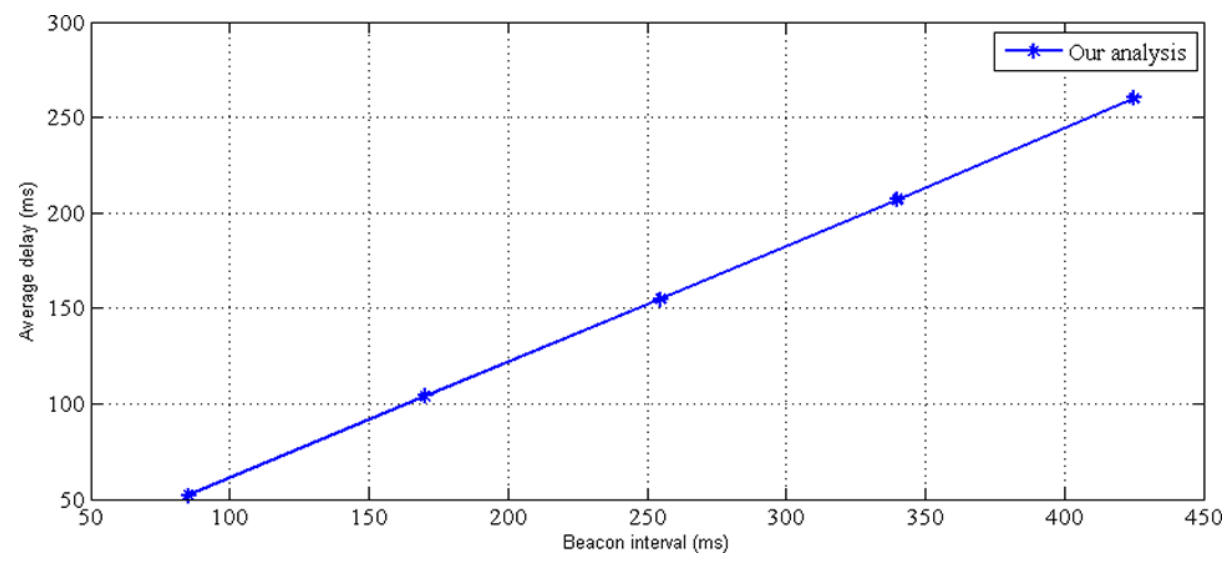

Fig. 9. Average buffered delays with the values of BI

Comparing QPSK Ro-FSO communication systems in terms of maximum distance signal can pass through the same atmosphere conditions (heavy fog) during its performances (Q.factor and BER). The single-channel could have the longest range than multi-channel, increasing transmission signal channels decreasing the transmission ranges. Although, the feature of the multi-channel system it's getting a higher data rate at the same time. 


\section{Conclusion}

It can be concluded from the literature of the architectures proposed in this research that this work encourages the development of modern communication systems. Although, this research was articulated the simulation models of Radiofrequency over Free Space optics (Ro-FSO) communication technique. A designed Ro-FSO system has investigated the radio frequency (RF) signals with various RF modulation techniques (ASK, QAM, and QPSK). The best system performance has been found by the QPSK modulation technique. For this reason, the RF modulation QPSK has been chosen to develop the wireless communication of the Ro-FSO system. Subsequently, WDM technology has been employed to transmit more than one signal to increase the transferred information capacity. Therefore, the 20 Gbps transported by two RF signals (20, and 50) $\mathrm{GHz}$ also has been transported $40 \mathrm{Gbps}$ data rate by four signals of RF $(20,30,40$, and 50) GHz. Finally, the WDM Ro-FSO system could transport $80 \mathrm{Gbps}$ where every $10 \mathrm{Gbps}$ placed on $20 \mathrm{GHz}$ and modulated with $(1552.5,1551.7,1550.9$, $1550.2,1549.3,1548.5,1547.7,1546.9) \mathrm{nm}$. Hence, the digital data rate can transmit along with various atmospheric weather conditions (clear, haze, rain, and fog) of FSO links. Despite the different values of RF signals were used in the Ro-FSO system, the higher RF $(50 \mathrm{GHz})$ has wider bandwidth to have larger information while the lower $\mathrm{RF}$ signal $(20 \mathrm{GHz})$ has transferred over long ranges when it has a low data rate.

\section{$9 \quad$ References}

[1] K. R. Patel, and S. K. Mulye, "Free Space Optics (FSO)-Past, Present, Future and Mathematical Models of Atmospheric Turbulence for FSO Link Budget Analysis," IJRITCC, 2016.

[2] P. Kumara, and R. Thakur, "Review Paper: Hybrid Amplifier in FSO System," International Journal of Computer Technique, vol. 3, no. 2, 2016.

[3] W.-S. Tsai, H.-H. Lu, C.-Y. Li, T.-C. Lu, H.-H. Lin, B.-R. Chen, and C.-J. Wu, "A 50 m/320Gbps DWDM FSO Communication with A Focal Scheme," IEEE Photonics, vol. 8, pp. 1943-0655, 2016. https://doi.org/10.1109/JPHOT.2016.2555618

[4] T. S. Mansour, and Fahad M. Abdulhussein, "Dual Measurements of Pressure and Temperature with Fiber Bragg Grating Sensor," Al-Khwarizmi Engineering Journal, vol. 11, no. 2, pp. 86-91, 2015.

[5] I. A. Aljazaery, H. T. Salim, and M. R. Aziz, "Combination of Hiding and Encryption for Data Security," International Journal of Interactive Mobile Technologies, vol. 14, no. 9, pp. 34-47, 2020. https://doi.org/10.3991/ijim.v14i09.14173

[6] O. H. Yahya, H. Salim, and I. Aljazaery, "Reducing the Data Rate in Internet of Things Applications by Using Wireless Sensor Network," International Journal of Online and Biomedical Engineering (iJOE), vol. 16, no. 3, pp. 107-116, 2020. https://doi.org/10.3991/ijoe. v16i03.13021

[7] X. Zhang, J. H. Cui, S. Das, M. Gerla, and M. Chitre, "Underwater Wireless Communications and Networks: Theory and Application: Part 2 [Guest Editorial]," IEEE Commun. Mag., vol. 54, no. 2, pp. 30-31, 2016. doi: https://doi.org/10.1109/MCOM.2016. 7402257

[8] M. Al-Rubaiai, "Design and Development of an LED-Based Optical Communication System,” Michigan State University, 2015. https://doi.org/10.1109/AIM.2016.7576760 
[9] P. A. McGillivary, V. Chirayath, and J. Baghdady, "Use of multi-spectral high repetition rate LED systems for high bandwidth underwater optical communications, and communications to surface and aerial systems," 2018 4th Underw. Commun. Netw. Conf. UComms 2018, pp. 1-5, 2018, doi: https://doi.org/10.1109/UComms.2018.8493228

[10] H. T. Alrikabi, A. H. M. Alaidi, A. S. Abdalrada, and F. T. Abed, "Analysis the Efficient Energy Prediction for 5G Wireless Communication Technologies," International Journal of Emerging Technologies in Learning (iJET), vol. 14, no. 8, pp. 23-37, 2019. https://doi. org/10.3991/ijet.v14i08.10485

[11] B. Majlesein, A. Gholami, and Z. Ghassemlooy, "A Complete Model for Underwater Optical Wireless Communications System," 2018 11th Int. Symp. Commun. Syst. Networks Digit. Signal Process. CSNDSP 2018, July, 2018, doi: https://doi.org/10.1109/ CSNDSP.2018.8471869

[12] I. A. Ibrahim, H. T. Salim, and H. F. Khazaal, "Investigating the Analysis of Power Saving Mode in IEEE 802.11 for Wi-Fi Communication," Wasit Journal of Engineering Sciences, vol. 6, no. 3, pp. 13-19, 2018. https://doi.org/10.31185/ejuow.Vol6.Iss3.100

[13] J. Xu et al., "Underwater Laser Communication Using an OFDM-Modulated 520-nm Laser Diode,” IEEE Photonics Technol. Lett., vol. 28, no. 20, pp. 2133-2136, 2016. https://doi. org/10.1109/LPT.2016.2582542

[14] S. Chaudhary, and A. Amphawn, " $4 \times 2.5 \mathrm{Gbps}=10 \mathrm{Gbps}$ RO-FSO Transmission system by Incorporating Hybrid WDM-MDM of spiral phased LG-HG Modes," International Conference on Internet Applications, Protocols and Services, pp. 978-967, 2015.

[15] C.-M. Ho et al., "A 10m/10Gbps underwater wireless laser transmission system," in Optical Fiber Communication Conference, 2017, pp. Th3C-3.

[16] H. T. S. Al-Rikabi, Enhancement of the MIMO-OFDM Technologies. California State University, Fullerton, 2013.

[17] T. Rahman, S. Iqbal, and M. Islam, "Modeling and Performance Analysis of Free Space Optical Communication System," International Conference on Infonnatics, Electronics and Vision, pp. 211-218, 2012. https://doi.org/10.1109/ICIEV.2012.6317371

[18] Md. S. Hossain, S. Howlader, and R. Basak, "Invistagation the Q.Factor and BER of a WDM System in Optical Fiber Communication Network by using SOA," International Journal of Innovation and Scientific Research, vol. 13, no. 1, pp. 315-322, 2015.

[19] P. Sharma, H. Sarangol, J. Malhota, "Determined Effect of Weather Conditions on FSO Based System Survey," International Journal of Application or Innovation in Engineering and Management, vol. 4, no. 6, pp. 74-79. 2015.

[20] M. Al-Dabag, H. Th. Salim ALRikabi, and R. Al-Nima, "Anticipating Atrial Fibrillation Signal Using Efficient Algorithm," International Journal of Online and Biomedical Engineering (iJOE), vol. 17, no. 2, pp. 106-120, 2021. https://doi.org/10.3991/ijoe. v17i02.19183

[21] A. S. Hussein, R. S. Khairy, S. M. M. Najeeb, and H. T. Salim, "Credit Card Fraud Detection Using Fuzzy Rough Nearest Neighbor and Sequential Minimal Optimization with Logistic Regression,” International Journal of Interactive Mobile Technologies, vol. 15, no. 5, 2021. https://doi.org/10.3991/ijim.v15i05.17173

[22] A. Mohammed, R. Chisab, and H. Salim, "Efficient RTS and CTS Mechanism Which Save Time and System Resources," International Journal of Interactive Mobile Technologies, vol. 14, no. 4, pp. 204-211, 2020. https://doi.org/10.3991/ijim.v14i04.13243

[23] N. A. Hussien, A. A. Daleh Al-Magsoosi, H. T. AlRikabi, and F. T. Abed, "Monitoring the Consumption of Electrical Energy Based on the Internet of Things Applications," International Journal of Interactive Mobile Technologies, vol. 15, no. 7, 2021. https://doi. org/10.3991/ijim.v15i07.20183 
[24] P. Singal, S. Rai, R. Punia, and D. Kashyap, "Comparison of Different Transmitter Using $1550 \mathrm{~nm}$ and $10000 \mathrm{~nm}$ in FSO Communication Systems," International Journal of Computer Science \& Information Technology (IJCSIT), vol. 7, no. 3, June 2015. https://doi. org/10.5121/ijcsit.2015.7309

[25] A. Amphawan, S. Chaudhary, and V. W. S. Chan, "2×20 Gbps-40 GHz OFDM Ro-FSO Transmission with Mode Division Multiplexing," Journal of European Optical Society Rapid Publication, vol. 9, no.14041, 2014. https://doi.org/10.2971/jeos.2014.14041

[26] Y. I. Hammadi, Tahreer S. Mansour, A. H. H. Al-Masoodi, and S. W. Harun, "Passively Femtosecond Mod- Locked Erbium-Doped Fiber Oscillator with External Pulse Compressors for Frequency Comb Generation," Journal of Optical Communication.

[27] I. A. Ibrahim, and Tahreer S. Mansour, "Walsh Hadamard Code and Wavelength Division Multiplexing Designed and Implemented Using Array FBGs,” IOP Conf. Ser... Mater. Sci. Eng. 1094 012114. 2021. https://doi.org/10.1088/1757-899X/1094/1/012114

\section{Authors}

Isam Aameer Ibrahim obtained his Bachelor's degree in Electrical Engineering from the College of Engineering, University of Mustansiriya. As well as obtained his Master's Degree in Communication Engineering from the College of Engineering, University of Uniten. Currently, he is studying his Ph.D. Degree in Communication and Electronics Engineering at the Institute of Laser for postgraduate studies at, University of Baghdad. His research interest area focuses on applications of Laser in Communication and Electronics Engineering. Email: isam.omair1101a@ilps.uobaghdad.edu.iq.

Tahreer S. Mansour was born in Baghdad, Iraq, on July 13, 1976. She has received the bachelor's degree from The Al-Nahrain University from the College of Communication and Electronics Engineering, Baghdad, Iraq, in 1998. She has received the master's degree from The Al-Nahrain University from the College of Communication and Electronics Engineering, Baghdad, Iraq, in 2001. She has received a Ph.D. from Baghdad University in laser institute for postgraduate studies, 2009. Design photonic devices to enhance the performance of optical communication systems is an area of interest.

Article submitted 2021-05-27. Resubmitted 2021-06-29. Final acceptance 2021-07-02. Final version published as submitted by the authors. 